\title{
Rheumatoid Arthritis and Risk of Sexual Dysfunction: A Systematic Review and Metaanalysis
}

\author{
Shankun Zhao, Ermao Li, Jiamin Wang, Lianmin Luo, Jintai Luo, and Zhigang Zhao
}

\begin{abstract}
Objective. It has been reported that there is an association between rheumatoid arthritis (RA) and increased susceptibility to sexual dysfunction (SD). This systematic review and metaanalysis aimed to investigate whether RA was a risk factor for SD.

Methods. MEDLINE (PubMed), EMBASE, and the Cochrane Library were systematically searched for all studies assessing sexual function in patients with RA. The association between RA and risk of SD was summarized using relative risk (RR) with $95 \%$ CI.

Results. Overall, 44,745 participants (mean age 43.2 yrs) were included from 7 studies (4 cross-sectional and 3 case-control studies). Of these, 6642 were patients with RA, with the mean disease duration from 5.7 years to 12.17 years. The methodological qualities of the included studies were judged as moderate to high. Synthesis of results demonstrated that RA was significantly associated with an increased risk of SD in females (RR 1.73, 95\% CI 1.36-2.22, p < 0.001; heterogeneity: $\mathrm{I}^{2} 60.3 \%, \mathrm{p}=0.028$ ) as well as in males (RR 1.99, 95\% CI 1.64-2.43, p < 0.001). The outcomes related to the Grading of Recommendations Assessment, Development, and Evaluation approach showed that the absolute effect of RA on SD was 10 more per 1000 (6-15 more); the overall quality of evidence was rated as low.

Conclusion. Evidence from included studies indicates that patients with RA have a significantly increased risk of SD, which suggests that both patients and clinicians should be aware of the potential role of RA in the development of SD. (First Release June 1 2018; J Rheumatol 2018;45:1375-82; doi:10.3899/jrheum.170956)
\end{abstract}

Key Indexing Terms:

RHEUMATOID ARTHRITIS SEXUALDYSFUNCTION RISK METAANALYSIS

Rheumatoid arthritis (RA) is an inflammatory disease characterized by the immune-mediated destruction of joints, which

From the Department of Urology and Andrology, Minimally Invasive Surgery Center, Guangdong Provincial Key Laboratory of Urology, The First Affiliated Hospital of Guangzhou Medical University, Guangzhou, Guangdong, China.

S. Zhao, MD, Department of Urology and Andrology, Minimally Invasive Surgery Center, Guangdong Provincial Key Laboratory of Urology, The First Affiliated Hospital of Guangzhou Medical University; E. Li, MD, Department of Urology and Andrology, Minimally Invasive Surgery Center, Guangdong Provincial Key Laboratory of Urology, The First Affiliated Hospital of Guangzhou Medical University; J. Wang, MD, Department of Urology and Andrology, Minimally Invasive Surgery Center, Guangdong Provincial Key Laboratory of Urology, The First Affiliated Hospital of Guangzhou Medical University; L. Luo, MD, Department of Urology and Andrology, Minimally Invasive Surgery Center, Guangdong Provincial Key Laboratory of Urology, The First Affiliated Hospital of Guangzhou Medical University; J. Luo, MD, PhD,

Department of Urology and Andrology, Minimally Invasive Surgery Center, Guangdong Provincial Key Laboratory of Urology, The First Affiliated Hospital of Guangzhou Medical University; Z. Zhao, MD, PhD, Department of Urology and Andrology, Minimally Invasive Surgery Center, Guangdong Provincial Key Laboratory of Urology, The First Affiliated Hospital of Guangzhou Medical University. Drs. S. Zhao, E. Li, and J. Wang contributed equally to this work.

Address correspondence to Dr. Z. Zhao, Department of Urology and Andrology, Minimally Invasive Surgery Center, Guangdong Provincial Key Laboratory of Urology, The First Affiliated Hospital of Guangzhou

Medical University, Guangzhou, Guangdong 510230, China.

E-mail:zgzhaodr@126.com

Accepted for publication February 15, 2018. is 2- to 3-fold more frequent in females than in males ${ }^{1,2}$. Epidemiological studies have indicated that RA affects an estimated $0.5-1 \%$ of the adult population ${ }^{3}$. RA is a systemic autoimmune disease, which often leads to diarthrodial joint destruction, decline in joint mobility, pain, and fatigue ${ }^{4}$. These physiological and anatomical impairments may influence the sexual health of patients with RA. In addition, the long and chronic course of the disease as well as the medications for the disease can also cause sexual problems in these patients ${ }^{5}$. Thus, an association between sexual dysfunction (SD) and patients with RA may exist.

$\mathrm{SD}$ is a widespread health problem worldwide in middle-aged and elderly men and women ${ }^{6}$. Based on epidemiological data from different geographical regions, the prevalence of SD ranged from $18.4 \%$ to $30 \%$ among men, and from $25.8 \%$ to $67 \%$ among women ${ }^{7,8,9,10}$. Male SD mainly includes erectile dysfunction (ED), diminished libido, and abnormal ejaculation ${ }^{11}$, while the 4 major categories of female (F-) SD are desire, arousal, orgasmic, and sexual pain disorders ${ }^{12}$. SD is a multifactorial condition that can be affected by biological, psychological, and interpersonal determinants ${ }^{13}$. Currently, a wide variety of risk factors have been confirmed for the development of SD. In addition to well-known independent risk factors such as aging, diabetes mellitus, cardiovascular disease, dyslipidemia, and depression ${ }^{14}$, other potential factors have gradually been

Personal non-commercial use only. The Journal of Rheumatology Copyright (c) 2018. All rights reserved. 
recognized as increasing susceptibility to SD. Over the past several years, a number of studies have addressed the relationship between $\mathrm{RA}$ and $\mathrm{SD}^{15}$. The proportion of patients with RA reporting SD ranged across studies from $37 \%$ to $66 \% \%^{1,4}$. However, reliable results have been inconsistent with this relationship. In the study by van Berlo, et al, male and female patients with RA did not experience more sexual problems than the control population ${ }^{16}$.

Although a trend toward a higher prevalence of SD in patients with RA was found, the evidence for the potential connection is controversial and the well-established information was limited. Our current review was performed in an attempt to investigate whether RA was a risk factor for SD. We elected to use a metaanalysis of the available studies to address this issue.

\section{MATERIALS AND METHODS}

We reported this systematic review and metaanalysis in accordance with the Preferred Reporting Items for Systematic reviews and Meta-Analyses (PRISMA) guidelines. The PRISMA checklist is shown in Supplementary Table 1 (available from the authors on request). The protocol for this systematic review is available from PROSPERO (CRD42017073586; www.crd.york.ac.uk/PROSPERO).

Data sources and searches. MEDLINE (PubMed), EMBASE, and the Cochrane Library were systematically searched from the inception of the databases to March 11,2017. The search was restricted to English language and human participants. We used the subject headings and the text keywords for the search. The search strategy used for MEDLINE included the following MeSH terms and text: ((“"Arthritis, Rheumatoid”[Mesh]) OR Rheumatoid Arthritis)) AND ((((((“Erectile Dysfunction”[Mesh]) OR sexual function) OR sexual dysfunction) OR "Sexual Dysfunctions, Psychological"[Mesh]) OR "Sexual Dysfunction, Physiological"[Mesh]) OR Impotence). Literature search, study selection, eligible studies quality assessment, and data extraction were performed by 2 authors (SZ and JW) independently. Disagreements were resolved by consensus or consultation with a third author (ZZ).

Measurement of RA and sexual dysfunction. Definitions of RA and SD were based on the International Classification of Diseases codes. Patients with RA were diagnosed on the basis of clinical symptoms and the clinical diagnosing criteria of the American College of Rheumatology. We included the studies in which SD was measured using any of the existing and validated instruments. These included questionnaires, physical examination, and clinical assessment of sexual function in the eligible studies. Sexual functionality in female subjects was assessed using either the Female Sexual Function Index (FSFI) or the Changes in Sexual Functioning Questionnaire, and women with a total score of $<26.5$ and 40 , respectively, were considered to have FSD.

Study selection. We included all the available epidemiologic evidence on the association between RA and risk of SD in adults. Participants in our present study were limited to the broad-spectrum population diagnosed with RA and $\mathrm{SD}$. On the basis of the Patient, Intervention, Comparison, Outcome and Study design (PICOS) framework, the following question guided this review: Does RA increase the risk of SD? The PICOS evidence base used consisted of the following combinations: SD in an adult patient (P); history of RA (I); comparison with the general population (C); diagnosis of SD, ED, FSD, frigidity $(\mathrm{O})$. We accepted all study designs (S). Further, studies on the pertinent subjects that provided relative risk (RR), HR estimates, OR with $95 \% \mathrm{CI}$, or adequate data to allow calculation of these efficiency measures were included. The exclusion criteria included the following: (1) unreported control data; (2) review articles, meeting abstracts, comments, editorials, letters, case reports, or congress reports; (3) duplicated or updated data;
(4) animal experiments; and (5) studies with $<10$ participants in either the exposure or control group. For multiple publications of the same clinical trial, only the most recent study was included.

Data extraction. Two authors independently extracted the following data: the first authors' names, year of publication, study regions, study design, sex, the case and control sample sizes, age, methods of RA and SD ascertainment, and statistical adjustments for confounding factors.

Quality assessment. The methodological quality assessment of the cross-sectional and case-control studies was based on the cross-sectional study quality methodology checklist and the Newcastle-Ottawa Scale (NOS) ${ }^{17,18,19}$, respectively. The Grading of Recommendations Assessment, Development, and Evaluation (GRADE) approach was applied to generate absolute estimates of the risk of SD in women and men with RA, and to evaluate and rank the overall quality of the evidence.

Statistical analyses. The strength of association between RA and the risk of SD in the included studies was estimated using RR and its 95\% CI (standard mean differences and 95\% CI were calculated for scores). Results with a 2 -tailed $\mathrm{p}$ value $<0.05$ were considered statistically significant in this analysis. The heterogeneity of studies was assessed using the $\mathrm{I}^{2}$ statistic and the Cochrane Q statistic ( $\mathrm{I}^{2}>50 \%$ was considered to be consistent with substantial heterogeneity; $p$ value of $Q$ test $<0.10$ was considered statistically significant). Because of the high likelihood of between-study variance for differences in study design and study population, a random effects model rather than a fixed effects model was applied.

Subgroup analyses were conducted to further explore the origin of heterogeneity. Sensitivity analysis was performed to determine the potential source of heterogeneity by omitting studies one by one and evaluating the resulting effect. Begg and Egger tests were used for publication bias analysis. The current statistical analysis was performed using Review Manager (version 5.3, The Cochrane Collaboration) and Stata (version 13.0, Stata Corp LP).

\section{RESULTS}

Literature search. Through a broad selection of the electronic databases, a total of 407 articles were identified in the initial search. Of these articles, 360 were excluded after removing duplicates and irrelevant articles, and reviewing the titles and abstracts. Forty-seven potentially relevant studies remained for further full-text review. Among them, 5 studies were excluded for giving an unclear definition of RA or SD; 14 for having no control group; 4 for inappropriate grouping; 7 for insufficient outcome data; and 8 for being reviews or comments. Full-text excluded studies with reasons for exclusion are shown in Supplementary Table 2 (available from the authors on request). Finally, this metaanalysis comprised 7 observational studies ${ }^{20-26}$ that met the inclusion criteria. The selection process is shown in Figure 1.

Study characteristics. The included studies were cross-sectional ${ }^{20,23,25,26}$ or case-control ${ }^{21,22,24}$ in design. Among all 7 studies, a total of 44,745 individuals were enrolled. Of these, 6642 were patients with RA, while the remaining 38,103 participants were healthy subjects. The mean disease duration of RA was 5.7-12.17 years. Six studies reported the prevalence of FSD; 1 study reported the prevalence of ED. The detailed characteristics of the included studies are summarized in Table 1.

Study quality and overall quality of the evidence. The results of the methodological quality assessment of the cross-sec-

Personal non-commercial use only. The Journal of Rheumatology Copyright $\odot$ (2018. All rights reserved 


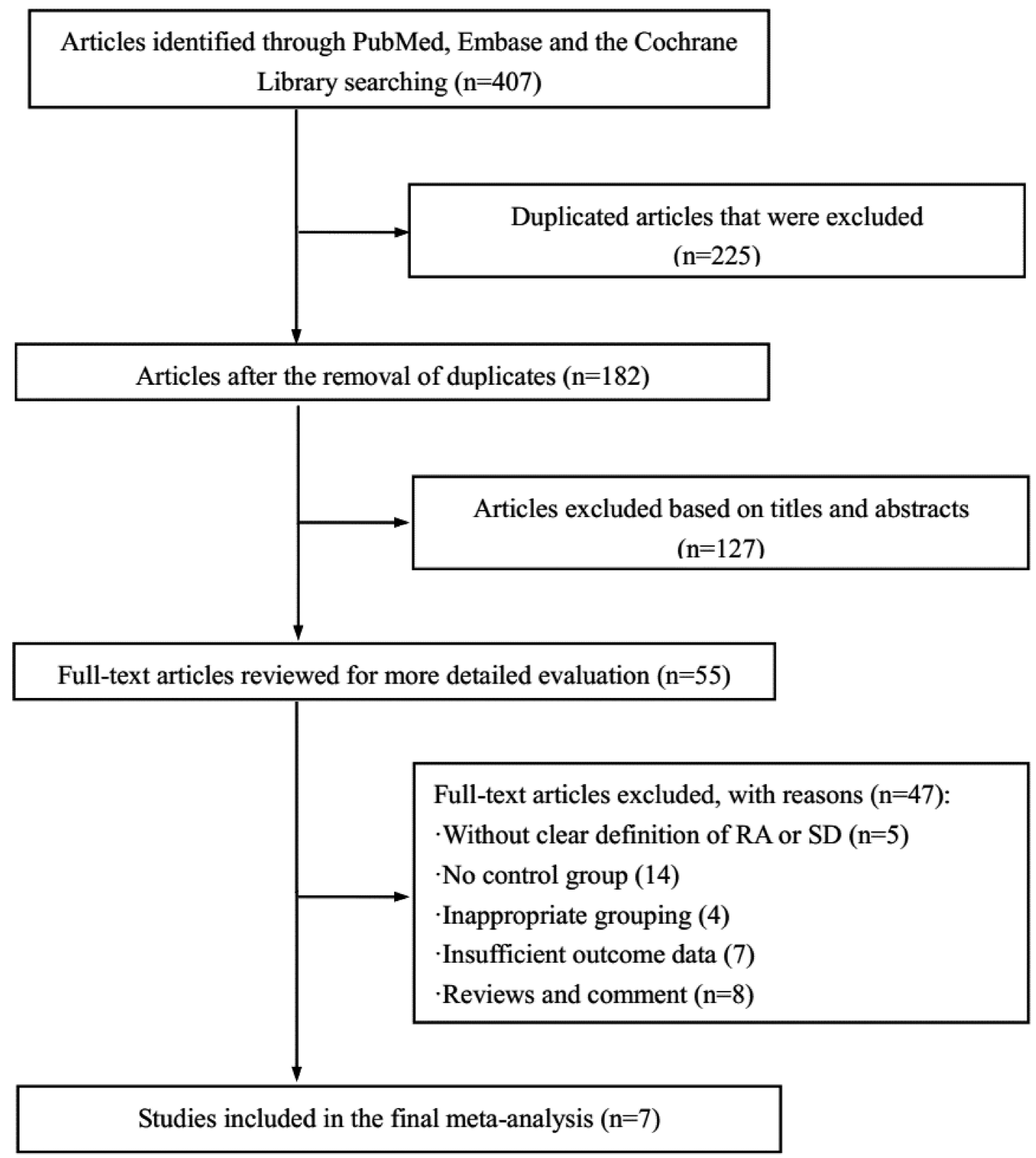

Figure 1. Flow chart of study selection. RA: rheumatoid arthritis; SD: sexual dysfunction.

tional studies are shown in Supplementary Table 3 (available from the authors on request). Two studies 26,27 were considered high quality and 2 studies $^{22,23}$ as moderate quality. Using the NOS, 1 study ${ }^{22}$ was evaluated to be high quality and 2 studies $^{21,24}$ medium quality (Supplementary Table 4, available from the authors on request). The GRADE-relevant outcomes showed that the rate of events of SD on average in patients with RA was 403/6642 (6.1\%), whereas it was $507 / 38,103(1.3 \%)$ in patients without RA; the absolute effect of RA on SD was 10 more per 1000 (from 6 more to 15 more); the overall quality of the evidence was judged as low (Supplementary Table 5, available from the authors on request).
Synthesis of results. As shown in Figure 2, synthesis of results demonstrated that RA was significantly associated with an increased risk of SD in females (RR 1.73, 95\% CI 1.36-2.22, $\mathrm{p}<0.001$; heterogeneity: $\left.\mathrm{I}^{2} 60.3 \%, \mathrm{p}=0.028\right)$, as well as in males (RR 1.99, 95\% CI 1.64-2.43, p < 0.001).

Subgroup analyses. To further elicit the association between RA and the risk of SD and explore the heterogeneity, subgroup analyses were performed based on study design, age, and mean disease duration of RA (Table 2). Subgroup analysis by study design revealed that case-control studies $^{21,22,24}$ (RR 1.94, 95\% CI 1.38-2.74) had a higher risk of SD compared with cross-sectional studies ${ }^{20,23,25,26}$ (RR $1.61,95 \%$ CI $1.23-2.10, \mathrm{p}<0.001)$. There was no significant 
Table 1. Characteristics of the included studies.

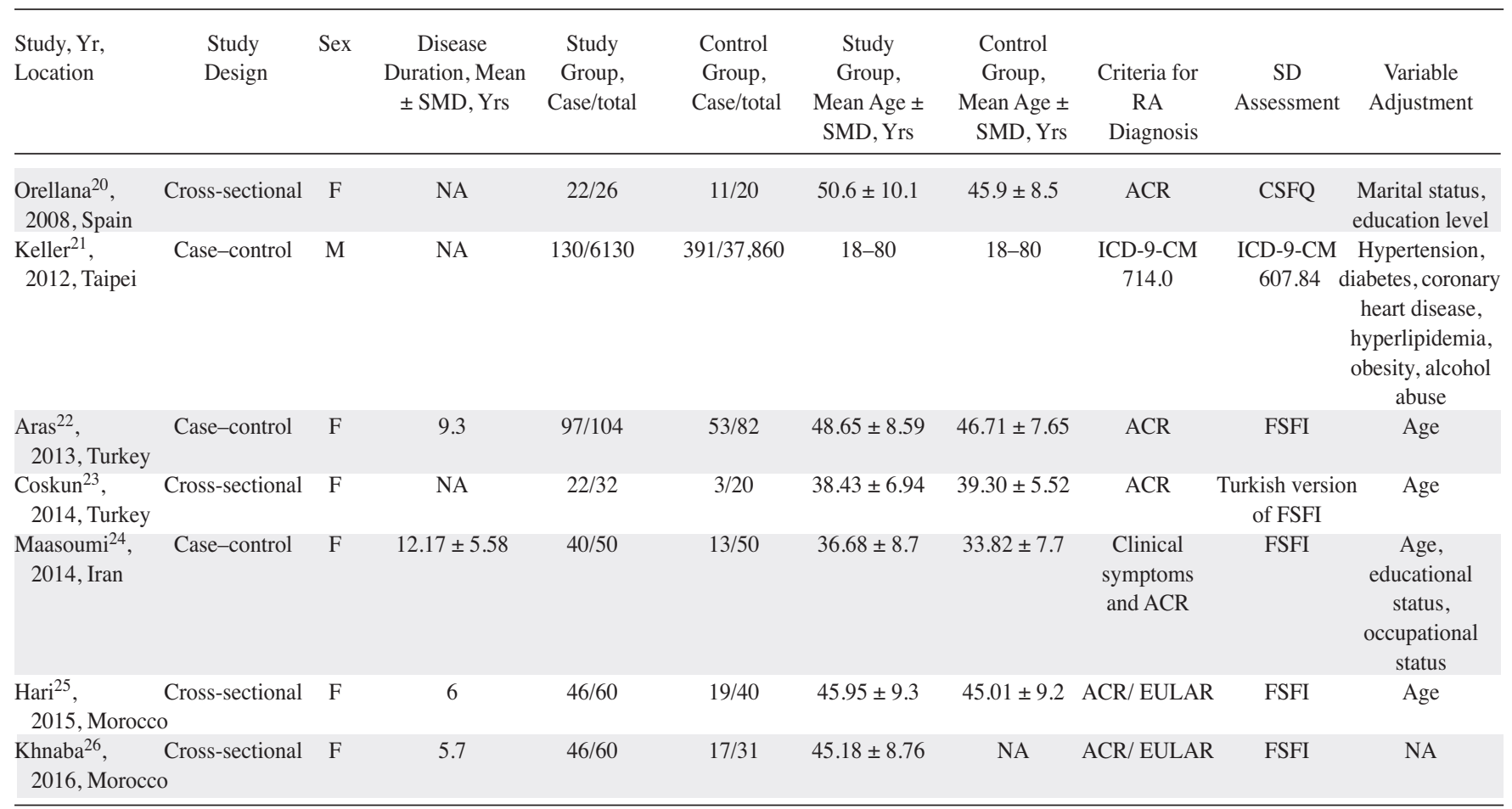

SMD: standard mean difference; RA: rheumatoid arthritis; SD: sexual dysfunction; NA: not available; ACR: American College of Rheumatology; ICD-9-CM: International Classification of Diseases-9-Clinical Modification; CSFQ: Changes in Sexual Functioning Questionnaire; EULAR: European League Against Rheumatism; FSFI: Female Sexual Function Index.

heterogeneity in cross-sectional studies $\left(\mathrm{I}^{2} 30.5 \%, \mathrm{p}=0.230\right)$, while substantial heterogeneity was detected in case-control studies $\left(\mathrm{I}^{2} 83.1 \%, \mathrm{p}=0.003\right)$. Stratified analysis by age showed that the association between age $<45$ years $^{23,24}$ and SD (RR 3.30, 95\% CI 2.11-5.14) was more significant for studies conducted in age $\geq 45$ years $^{20,22,25,26}$ (RR $1.47,95 \%$ CI $1.29-1.68, \mathrm{p}<0.001)$. No substantial heterogeneity was detected in either age subgroup $\left(\mathrm{I}^{2} 0.0 \%, \mathrm{p}=0.506\right.$ and $\mathrm{I}^{2} 0.0 \%, \mathrm{p}=0.933$, respectively). The relationship between mean disease duration and the risk of SD was reported in 4 studies. A stronger association was detected in groups with a duration $\geq 8$ years $^{22,24}(\mathrm{RR} 2.03,95 \%$ CI $0.97-4.25)$ compared with a duration $<8$ years $^{25,26}$ (RR $1.50,95 \%$ CI 1.17-1.92, $\mathrm{p}<0.001)$.

Because the samples of most included studies had female participants, we further explored the specific FSD domains on the basis of FSFI questionnaire within the studies. In combing through 4 relevant studies $22,23,24,25$ that provided the FSFI scores, we found that women with RA had significantly lower values in desire, arousal, lubrication, orgasm, satisfaction, pain, and total FSFI score compared with healthy controls, particularly in the orgasm dimension (Table 3).

Sensitivity analysis. Sensitivity analysis was performed to assess the influence of each individual study on the overall risk of SD. There was no substantial change on the overall combined RR, which ranged from 1.65 (95\% CI 1.37-1.99) to 1.89 (95\% CI 1.49-2.40) after excluding any of the studies (Table 4 and Supplementary Figure 1, available from the authors on request). Moreover, similar heterogeneity was obtained after each exclusion, with the $\mathrm{I}^{2}$ ranging from $52 \%$ to $70 \%$. The outcome indicated that no single study dominated the combined RR and heterogeneity.

Publication bias. Both Begg rank correlation test and Egger linear regression yielded nonsignificant publication bias among the studies (Begg, $\mathrm{p}>|\mathrm{z}|=0.230$; Egger, $\mathrm{p}>$ $|\mathrm{t}|=0.256,95 \% \mathrm{CI}-1.68$ to 5.02; Supplementary Figure 2, available from the authors on request).

\section{DISCUSSION}

In recent years, several epidemiological studies have examined the association between RA and risk of SD but provided inconsistent results. Our current study aimed to summarize all the available epidemiological evidence on the association between RA and SD in adults. Based on data from 4 cross-sectional and 3 case-control studies, our present study indicated that the proportion of SD was significantly higher among patients with RA than the control group. Women and men with RA might have more than $73 \%$ and $99 \%$ increased prevalence of SD than those without RA, respectively. These results were consistent with a previous study by El Miedany, et $a l^{5}$, who recruited 231 patients with RA and found that both men $(49 / 91,53.8 \%)$ and women $(64 / 140,45.7 \%)$ had RA and 


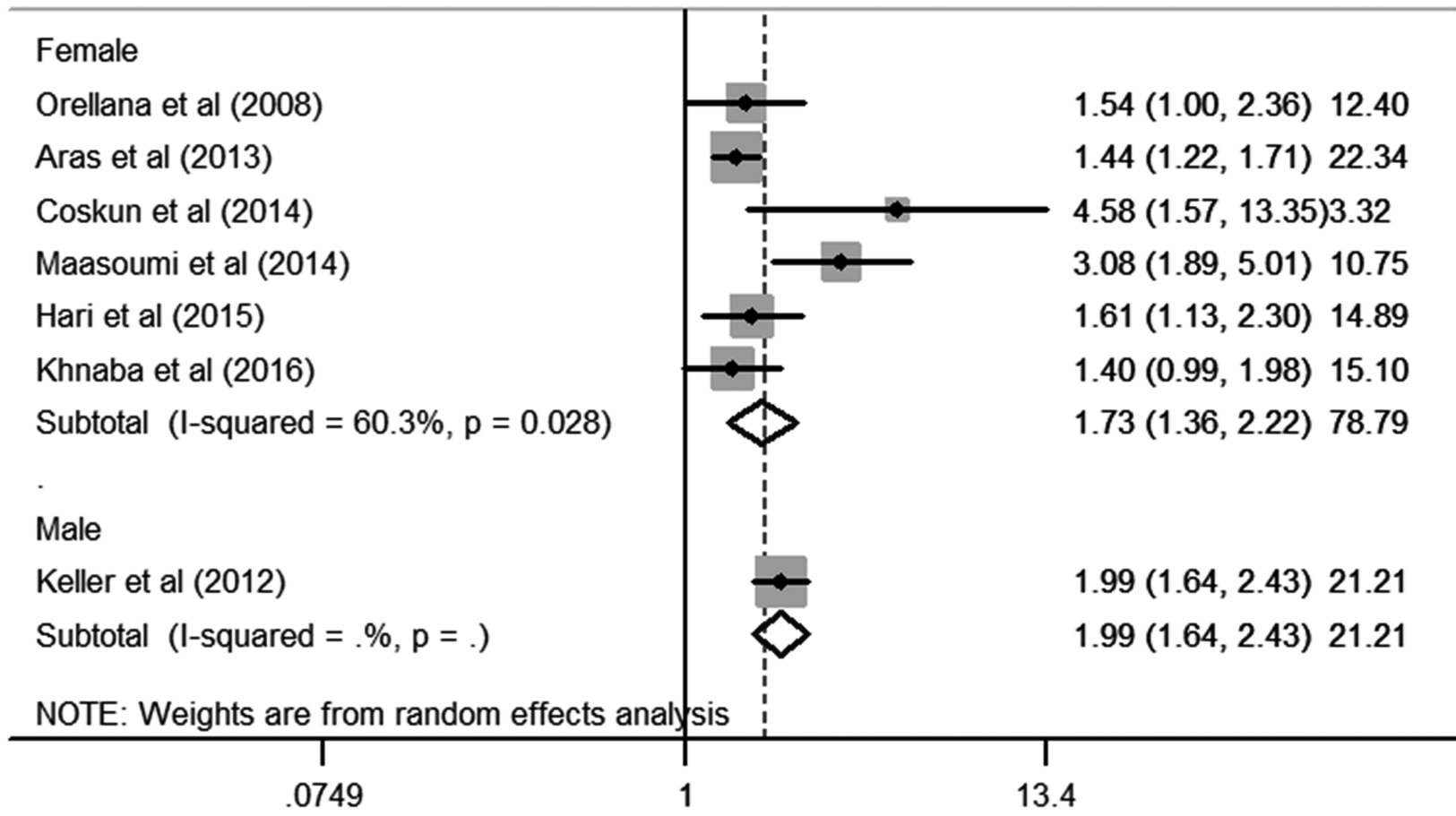

Figure 2. Forest plots of metaanalysis of the included studies on the association between RA and SD. RA: rheumatoid arthritis; SD: sexual dysfunction; RR: relative risk.

Table 2. Subgroup analysis of the association between RA and SD.

\begin{tabular}{|c|c|c|c|c|c|}
\hline \multirow[t]{2}{*}{ Variables } & \multirow[t]{2}{*}{ No. Studies } & \multicolumn{2}{|c|}{ Heterogeneity } & \multirow[t]{2}{*}{ RR (95\% CI) } & \multirow[t]{2}{*}{$\mathrm{p}$} \\
\hline & & $\mathrm{I}^{2}, \%$ & $\mathrm{p}$ & & \\
\hline \multicolumn{5}{|l|}{ Study design } & \multirow[t]{3}{*}{$<0.001$} \\
\hline Cross-sectional & 4 & 30.5 & 0.230 & $1.61(1.23-2.10)$ & \\
\hline Case-control & 3 & 83.1 & 0.003 & $1.94(1.38-2.74)$ & \\
\hline \multicolumn{5}{|l|}{ Age, yrs } & \multirow[t]{3}{*}{$<0.001$} \\
\hline$<45$ & 2 & 0.0 & 0.506 & $3.30(2.11-5.14)$ & \\
\hline$\geq 45$ & 4 & 0.0 & 0.933 & $1.47(1.29-1.68)$ & \\
\hline \multicolumn{5}{|c|}{ Mean disease duration, yrs } & \multirow[t]{3}{*}{$<0.001$} \\
\hline$<8$ & 2 & 0.0 & 0.571 & $1.50(1.17-1.92)$ & \\
\hline$\geq 8$ & 2 & 87.9 & 0.004 & $2.03(0.97-4.25)$ & \\
\hline
\end{tabular}

RA: rheumatoid arthritis; SD: sexual dysfunction; RR: relative risk.

SD. We also performed subgroup analyses to explore the degree to which potential confounders might have influenced the findings. When the sensitivity analyses were conducted, the quantification of the risk for SD remained significantly higher in nearly all the studies. However, substantial heterogeneity was also observed in our present study. The different sex, study design, age, mean disease duration, and varied characteristics of participants could all be partly responsible for this heterogeneity.

RA is a chronic inflammatory autoimmune disease that can lead to various degrees of disability and ultimately has a deleterious effect on sexual aspects of the patient's life, such as $\mathrm{SD}$. Baldursson and Brattstrom ${ }^{27}$ were the first to describe the association between sexuality problems and RA in both women and men. They found a high prevalence (63.6\%) of SD in patients with RA, and indicated that hip pain might be the main cause ${ }^{27}$. Moreover, studies showed that SD seems more closely associated with the severity of RA. Hill, et $a l^{28}$ revealed that $83 \%$ of the RA patients with functional capacity I or II were having sexual intercourse regularly, compared 
Table 3. Dimensions analysis of female sexual function on the basis of FSFI.

\begin{tabular}{lcccc}
\hline Dimensions & Heterogeneity & $\mathrm{S}$ & $\mathrm{SMD}(95 \% \mathrm{CI})$ & $\mathrm{p}$ \\
& $\mathrm{I}^{2}, \%$ & $\mathrm{p}$ & & \\
\hline Desire & 42.5 & 0.157 & $-1.17(-1.38$ to -0.97$)$ & $<0.001$ \\
Arousal & 87.5 & $<0.001$ & $-1.02(-1.62$ to -0.41$)$ & 0.001 \\
Lubrication & 74.3 & 0.009 & $-1.02(-1.44$ to -0.61$)$ & $<0.001$ \\
Orgasm & 79.8 & 0.002 & $-1.31(-1.80$ to -0.82$)$ & $<0.001$ \\
Satisfaction & 84.1 & $<0.001$ & $-1.24(-1.78$ to -0.69$)$ & $<0.001$ \\
Pain & 89.2 & $<0.001$ & $-0.76(-1.38$ to -0.13$)$ & 0.018 \\
Total score & 87.9 & 0.004 & $-1.15(-1.35$ to -0.94$)$ & $<0.001$ \\
\hline
\end{tabular}

FSFI: Female Sexual Function Index; SMD: standard mean difference.

Table 4. Sensitivity analysis after each study was excluded by turns.

\begin{tabular}{|c|c|c|c|}
\hline \multirow[t]{2}{*}{ Study Excluded } & \multirow[t]{2}{*}{ RR $(95 \% \mathrm{CI})$ for Remainders } & \multicolumn{2}{|c|}{ Heterogeneity } \\
\hline & & $\mathrm{I}^{2}, \%$ & $\mathrm{p}$ \\
\hline Orellana, et $a l^{20}$ & $1.72(1.44-2.32)$ & 70 & 0.006 \\
\hline Keller, et $a l^{21}$ & $1.73(1.36-2.22)$ & 60 & 0.03 \\
\hline Aras, et $a l^{22}$ & $1.89(1.49-2.40)$ & 56 & 0.05 \\
\hline Coskun, et $a l^{23}$ & $1.72(1.41-2.09)$ & 62 & 0.02 \\
\hline Maasoumi, et a $2^{24}$ & $1.65(1.37-1.99)$ & 52 & 0.06 \\
\hline Hari, et $a l^{25}$ & $1.82(1.43-2.33)$ & 70 & 0.005 \\
\hline Khnaba, et $a l^{26}$ & $1.87(1.47-2.37)$ & 68 & 0.009 \\
\hline
\end{tabular}

RR: relative risk.

with $60 \%$ with functional capacity III or IV. Gutweniger, et $a l^{29}$ also found that patients with high prevalence of RA symptoms had significantly more problems in sexuality than those with low prevalence.

FSD is a common health problem that severely harms quality of life and interpersonal relationships of patients. In female patients with RA, SD can appear before sexual activity (limiting arousal and fantasies), during intercourse (causing pain, limitations in mobility), and after sexual activity (decreasing satisfaction, increasing pain) ${ }^{30}$. In our current study, dimension analysis on the basis of FSFI showed that female patients with RA scored lower in each domain of sexual function than healthy controls, particularly in the orgasm dimension. Our findings were consistent with a previous metaanalysis that analyzed the effect of RA on female sexual function domains by using FSFI. However, their results showed that the lubrication dimension was the most affected in patients with $\mathrm{RA}^{31}$. In male subjects, the effect of RA on SD had also been extensively investigated. Studies reported that increased incidence of ED was found in the male patients with RA as compared with normal volunteers ${ }^{5}$.

In the subgroup analysis on age, we found that more significant association was detected in the age group $<45$ years compared with $\geq 45$ years. It is well known that old age is an independent risk factor for SD. As women get older, age can provoke dyspareunia and decrease libido because of the reduction of hormone production. However, previous epidemiologic studies also found a high prevalence rate of SD among the young age group ${ }^{32}$. The possible etiological factors for SD in young people include drug use, poor mental health, smoking, and chronic conditions. Though most of the patients with RA were middle-aged or older ( $\geq 45$ yrs), 15 studies provided evidence that younger people with RA also had a remarkable prevalence of SD. Abdel-Nasser and Ali ${ }^{33}$ investigated 52 female patients with RA and found that $>60 \%$ of patients (mean age $39.8 \mathrm{yrs}$ ) experienced variable degrees of SD. Moreover, it was suggested that the importance of sexual health in patients with RA decreased with increasing age $^{34}$. Therefore, it seems reasonable to suggest that patients with RA at a relatively younger age are at high risk for SD.

Stratified analysis by mean disease duration in our study showed that duration $\geq 8$ years had a higher risk of SD compared with $<8$ years. Similarly, Karlsson, et al ${ }^{35}$ reported that patients with early RA $(<2$ yrs $)$ were more satisfied with their sexual life compared with patients with longstanding disease (20-24 yrs). Lin, et $a l^{1}$ also found that SD was more common in women with a longer duration of RA (mean duration 9.72 yrs).

Although many available clinical studies have linked RA to SD in both sexes, no clear-cut etiological and pathogenic mechanism has been identified to explain this positive association. The potential pathogenesis of increased SD symptoms in female patients with RA may be attributed to several factors. First, studies attributed FSD in RA to several disease-related physiological disorders such as restrictions on joint mobility, morning stiffness, pain, and fatigue ${ }^{4,15}$. RA patients with pain often experience a decrease in sexual desire, difficulties in sexual positions, and a lower rate of $\operatorname{orgasms}^{36}$. Fatigue impairs sexual activity by decreasing sexual drive and interfering with sexual intercourse ${ }^{37}$. Other common symptoms of RA, such as limitations of joint mobility and morning stiffness, could also impede sexual function because of uncomfortable conditions during sexual activities $^{16,33,38}$. Second, psychological morbidities such as depression, low self-esteem, anxiety, negative body image perception, and worries about partner interest are often observed among female patients with RA, and these variables are consistently found to be risk factors for SD $29,39,40$. A previous study ${ }^{5}$ suggested that SD symptoms might be related to psychological and sociocultural effects of RA. It

Personal non-commercial use only. The Journal of Rheumatology Copyright @ 2018. All rights reserved 
was also reported that depression was significantly correlated with pain ${ }^{41}$. Therefore, there might be a vicious circle of pain, depression, and SD in patients with RA. Some researchers even concluded that psychological distress was more important than objectively assessed disease status in determining sexual satisfaction in patients with $\mathrm{RA}^{33,42}$. Third, hormonal imbalance has also been suggested as potentially involved in the relationship between RA and SD. Disturbances of sex hormone status can induce SD. It was reported that female patients with RA have a reduction in levels of androgen, such as testosterone, dihydrotestosterone, and dehydroepiandrosterone (DHEA) ${ }^{43}$. A study by Labrie, et $a l^{44}$ indicated that reduction of DHEA might be a risk factor for dyspareunia and vaginal dryness. The potential mechanisms of association between RA and male SD shared similar pathological factors as those in females, such as pain, fatigue, joint mobility, depression, and hypoandrogenicity $5,40,45,46$.

Since RA was associated with SD risk, the establishment of an optimal treatment strategy to improve the sexual health of patients with RA is warranted. The treatment of SD will depend on the specific patient's symptoms. Accumulated data from both epidemiologic and clinical studies have suggested that physiotherapy, psychotherapy, and medical treatments may offer a useful intervention for treating comorbid SD in patients with RA. The recommendations of physiotherapy include mobility treatment, pain reduction, physical activities, and increased endurance. Improved daily functional capacity, reduced pain, and regular physical activity can raise sexual intercourse frequency and psychological well-being ${ }^{30}$. In addition, Brodin, et $a l^{47}$ reported that increased physical activity levels can reduce fatigue and increase perceived health status for patients with RA. Further, heat and muscle relaxants before sexual activity might be beneficial to alleviate pain and sexual difficulties ${ }^{17}$. It has also been shown that total hip replacement can improve sexual disability to pre-disease levels in patients with RA (total effective rate reached $50 \%)^{48}$. Psychotherapies were also considered an effective way for improving sexual health in patients with RA. Referrals to physicians or health professionals, educational programs, and psychological consultation regarding SD for both patients with RA and their partners may be useful ${ }^{37}$. Some studies revealed that SD was correlated with disease activity variables of RA ${ }^{25}$. Pharmacotherapy (such as antiinflammatory drugs and analgesics) in RA may be beneficial in controlling disease activity ${ }^{5,40}$. However, it is noteworthy that some researchers believe that not only increased disease activity but also medical treatment of RA harms sexual function, causing reduced sexual desire, depressed mood, and fear of $\operatorname{sex}^{25,49}$. Because the pathogenesis of SD in patients with RA is attributed to different factors, such as physiological disorders, psychological morbidities, hormonal imbalance, and medications, a better alternative in management for those patients should depend on their chief complaint and specific symptoms.
To our knowledge, this is the first systematic review and metaanalysis to explore the potential association between RA and SD in both sexes. Our results suggest that it is important to recognize the adverse effects of RA on SD. However, there were some inherent limitations to our research. First, as with any systematic review and metaanalysis, substantial heterogeneity across included studies was observed. We have performed subgroup analysis and sensitivity analyses to explore the potential causes for the observed heterogeneity. Second, though most of the included studies were adjusted for known potential causes of SD, other confounding factors within each study could modulate the SD risk. Third, there was an absence of information derived from studies performed in the United States; there was also only a single included study evaluating SD in males with RA. In the future, high-quality randomized controlled trials with large sample sizes (especially in North and South American countries and for males) will be required, to validate the evidence of RA predisposing to the development of SD.

The results of this metaanalysis indicate a potentially hazardous effect of RA for developing SD in both women and men; however, further well-designed studies are still warranted to confirm this relationship. Given its association with SD, the assessment of sexual function as well as specific treatments are necessary for patients with RA in clinical practice.

\section{REFERENCES}

1. Lin MC, Lu MC, Livneh H, Lai NS, Guo HR, Tsai TY. Factors associated with sexual dysfunction in Taiwanese females with rheumatoid arthritis. BMC Womens Health 2017;17:12.

2. Kato E, Sawada T, Tahara K, Hayashi H, Tago M, Mori H, et al. The age at onset of rheumatoid arthritis is increasing in Japan: a nationwide database study. Int J Rheum Dis 2017;20:839-45.

3. Gabriel SE, Michaud K. Epidemiological studies in incidence, prevalence, mortality, and comorbidity of the rheumatic diseases. Arthritis Res Ther 2009;11:229.

4. Josefsson KA, Gard G. Sexual health in patients with rheumatoid arthritis: experiences, needs and communication with health care professionals. Musculoskeletal Care 2012;10:76-89.

5. El Miedany Y, El Gaafary M, El Aroussy N, Youssef S, Ahmed I. Sexual dysfunction in rheumatoid arthritis patients: arthritis and beyond. Clin Rheumatol 2012;31:601-6.

6. Lewis RW, Fugl-Meyer KS, Corona G, Hayes RD, Laumann EO, Moreira ED Jr, et al. Definitions/epidemiology/risk factors for sexual dysfunction. J Sex Med 2010;7:1598-607.

7. Selvin E, Burnett AL, Platz EA. Prevalence and risk factors for erectile dysfunction in the US. Am J Med 2007;120:151-7.

8. Corona G, Lee DM, Forti G, O’Connor DB, Maggi M, O'Neill TW; EMAS Study Group. Age-related changes in general and sexual health in middle-aged and older men: results from the European Male Ageing Study (EMAS). J Sex Med 2010;7:1362-80.

9. Ishak IH, Low WY, Othman S. Prevalence, risk factors, and predictors of female sexual dysfunction in a primary care setting: a survey finding. J Sex Med 2010;7:3080-7.

10. Cabral PU, Canario AC, Spyrides MH, Uchoa SA, Eleuterio JJ, Goncalves AK. Determinants of sexual dysfunction among middle-aged women. Int J Gynaecol Obstet 2013;120:271-4.

11. Kligman EW. Office evaluation of sexual function and complaints. Clin Geriatr Med 1991;7:15-39.

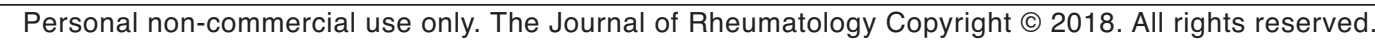


12. Angel K. The history of 'Female Sexual Dysfunction' as a mental disorder in the 20th century. Curr Opin Psychiatry 2010;23:536-41.

13. Hatzichristou D, Kirana PS, Banner L, Althof SE, LonneeHoffmann RA, Dennerstein L, et al. Diagnosing sexual dysfunction in men and women: sexual history taking and the role of symptom scales and questionnaires. J Sex Med 2016;13:1166-82.

14. Camacho ME, Reyes-Ortiz CA. Sexual dysfunction in the elderly: age or disease? Int J Impot Res 2005; 17 Suppl 1:S52-S56.

15. Shahar MA, Hussein H, Sidi H, Shah SA, Mohamed Said MS. Sexual dysfunction and its determinants in Malaysian women with rheumatoid arthritis. Int J Rheum Dis 2012;15:468-77.

16. van Berlo WT, van de Wiel HB, Taal E, Rasker JJ, Weijmar SW, van Rijswijk MH. Sexual functioning of people with rheumatoid arthritis: a multicenter study. Clin Rheumatol 2007;26:30-8.

17. Zeng X, Zhang Y, Kwong JS, Zhang C, Li S, Sun F, et al. The methodological quality assessment tools for preclinical and clinical studies, systematic review and meta-analysis, and clinical practice guideline: a systematic review. J Evid Based Med 2015;8:2-10.

18. Whiting P, Rutjes AW, Reitsma JB, Bossuyt PM, Kleijnen J. The development of QUADAS: a tool for the quality assessment of studies of diagnostic accuracy included in systematic reviews. BMC Med Res Methodol 2003;3:25.

19. Stang A. Critical evaluation of the Newcastle-Ottawa scale for the assessment of the quality of nonrandomized studies in meta-analyses. Eur J Epidemiol 2010;25:603-5.

20. Orellana C, Casado E, Masip M, Galisteo C, Gratacos J, Larrosa M. Sexual dysfunction in fibromyalgia patients. Clin Exp Rheumatol 2008;26:663-6

21. Keller JJ, Lin HC. A population-based study on the association between rheumatoid arthritis and erectile dysfunction. Ann Rheum Dis 2012;71:1102-3.

22. Aras H, Aras B, Icagasioglu A, Yumusakhuylu Y, Kemahli E, Haliloqlu S, et al. Sexual dysfunction in women with rheumatoid arthritis. Med Glas 2013;10:327-31.

23. Coskun B, Coskun BN, Atis G, Ergenekon E, Dilek K. Evaluation of sexual function in women with rheumatoid arthritis. Urol J 2014;10:1081-7.

24. Maasoumi R, Moridi M, Farhadi F, Moshfeghi Z. Sexual function in women with rheumatoid arthritis. Women Health Bull 2014;1:e20098.

25. Hari A, Rostom S, Lahlou R, Bahiri R, Hajjaj-Hassouni N. Sexual function in Moroccan women with rheumatoid arthritis and its relationship with disease activity. Clin Rheumatol 2015;34:1047-51 .

26. Khnaba D, Rostom S, Lahlou R, Bahiri R, Abouqal R, Hajjaj-Hassouni N. Sexual dysfunction and its determinants in Moroccan women with rheumatoid arthritis. Pan Afr Med J 2016;24:16.

27. Baldursson $\mathrm{H}$, Brattstrom H. Sexual difficulties and total hip replacement in rheumatoid arthritis. Scand J Rheumatol 1979; 8:214-6.

28. Hill RH, Herstein A, Walters K. Juvenile rheumatoid arthritis: follow-up into adulthood - medical, sexual and social status. Can Med Assoc J 1976;114:790-6.

29. Gutweniger S, Kopp M, Mur E, Gunther V. Body image of women with rheumatoid arthritis. Clin Exp Rheumatol 1999;17:413-7.

30. Areskoug-Josefsson K, Oberg U. A literature review of the sexual health of women with rheumatoid arthritis. Musculoskeletal Care 2009;7:219-26

31. Zhang Q, Zhou C, Chen H, Zhao Q, Li L, Cui Y, et al. Rheumatoid arthritis is associated with negatively variable impacts on domains of female sexual function: evidence from a systematic review and meta-analysis. Psychol Health Med 2018;23:114-25.

32. Akre C, Berchtold A, Gmel G, Suris JC. The evolution of sexual dysfunction in young men aged 18-25 years. J Adolesc Health 2014;55:736-43.

33. Abdel-Nasser AM, Ali EI. Determinants of sexual disability and dissatisfaction in female patients with rheumatoid arthritis. Clin Rheumatol 2006;25:822-30.

34. Hill J, Bird H, Thorpe R. Effects of rheumatoid arthritis on sexual activity and relationships. Rheumatology 2003;42:280-6.

35. Karlsson B, Berglin E, Wallberg-Jonsson S. Life satisfaction in early rheumatoid arthritis: a prospective study. Scand J Occup Ther 2006;13:193-9.

36. Saadat SH, Ramezani A, Ahmadi K. Sexual self-concept and general health in rheumatoid arthritis patients. Iran Red Crescent Med J 2015;17:e19005.

37. Ferreira CC, Da ML, Oliveira AC, de Carvalho JF, Lima RA, Simaan CK, et al. Frequency of sexual dysfunction in women with rheumatic diseases. Rev Bras Reumatol 2013;53:35-46.

38. Josefsson KA, Gard G. Women's experiences of sexual health when living with rheumatoid arthritis - an explorative qualitative study. BMC Musculoskelet Disord 2010;11:240.

39. Yilmaz H, Polat HA, Yilmaz SD, Erkin G, Kucuksen S, Salli A, et al. Evaluation of sexual dysfunction in women with rheumatoid arthritis: a controlled study. J Sex Med 2012;9:2664-70.

40. Tristano AG. The impact of rheumatic diseases on sexual function. Rheumatol Int 2009;29:853-60.

41. Abdel-Nasser AM, Abd ES, Taal E, El-Badawy SA, Rasker JJ, Valkenburg HA. Depression and depressive symptoms in rheumatoid arthritis patients: an analysis of their occurrence and determinants. Br J Rheumatol 1998;37:391-7.

42. van Lankveld W, Ruiterkamp G, Naring G, de Rooij DJ. Marital and sexual satisfaction in patients with RA and their spouses. Scand J Rheumatol 2004;33:405-8.

43. Gerosa M, De Angelis V, Riboldi P, Meroni PL. Rheumatoid arthritis: a female challenge. Womens Health 2008;4:195-201.

44. Labrie F, Archer DF, Koltun W, Vachon A, Young D, Frenette L, et al; VVA Prasterone Research Group. Efficacy of intravaginal dehydroepiandrosterone (DHEA) on moderate to severe dyspareunia and vaginal dryness, symptoms of vulvovaginal atrophy, and of the genitourinary syndrome of menopause. Menopause 2016;23:243-56.

45. Ostensen $\mathrm{M}$. New insights into sexual functioning and fertility in rheumatic diseases. Best Pract Res Clin Rheumatol 2004;18:219-32.

46. Perdriger A, Solano C, Gossec L. Why should rheumatologists evaluate the impact of rheumatoid arthritis on sexuality? Joint Bone Spine 2010;77:493-5.

47. Brodin N, Eurenius E, Jensen I, Nisell R, Opava CH. Coaching patients with early rheumatoid arthritis to healthy physical activity: a multicenter, randomized, controlled study. Arthritis Rheum 2008;59:325-31

48. Yoshino S, Fujimori J, Morishige T, Uchida S. Bilateral joint replacement of hip and knee joints in patients with rheumatoid arthritis. Arch Orthop Trauma Surg 1984;103:1-4.

49. Tasiemski T, Angiaszwili-Biedna N, Wilski M. Assessment of objective and subjective quality of life in people with rheumatoid arthritis - preliminary study. Ortop Traumatol Rehabil 2009: 11:346-59. 